\title{
Costos por corrosión de hierro ASTM A36 en procesos hidrometalurgicos auriferos. Comparación entre cianuro y
} tiourea

ReCIBIDO: 22/02/2017 AcEPTADO: 17/05/2017
En esta investigación se determinó la velocidad de corrosión (Vcorr) de hierro ASTM A36, en soluciones de cianuro de sodio en concentraciones de $0,62 \mathrm{~g} / \mathrm{L} ; 0,80$ $\mathrm{g} / \mathrm{L}$ y de tiourea $0.96 \mathrm{~g} / \mathrm{L} ; 1.24 \mathrm{~g} / \mathrm{L}$. Adicionalmente se evaluó el efecto de este deterioro en los costos asociados a procesos de lixiviación aurífera. La velocidad de corrosión se determinó en mm/año mediante curvas de polarización. Se realizó un análisis de costos utilizando indicadores sintéticos. Los resultados muestran que la velocidad de corrosión del metal es ligeramente mayor en la solución de tiourea 1,24 g/L (3,03 mm/año) con el siguiente comportamiento: corrosión de hierro ASTM A36 en Tiourea $1,24 \mathrm{~g} / \mathrm{L}>$ Tiourea $0,96 \mathrm{~g} / \mathrm{L}>$ Cianuro $0,80 \mathrm{~g} / \mathrm{L}$ $\approx$ Cianuro $0,62 \mathrm{~g} / \mathrm{L}$. Se pudo estimar que los costos por corrosión en los procesos de lixiviación aurífera se encuentran entre el 4,78 y $5,20 \%$ del capital para cianuro y tiourea respectivamente.

Palabras clave: costos, corrosión, acero ASTM A36, procesos hidrometalúrgicos.

COSTS BY ASTM A36 IRON CORROSION IN HYDROMETALURGIC AURIFEROUS PROCESSES. COMPARISON BETWEEN CYANIDE AND THIOUREA

\section{ABSTRACT}

The objective of this research was to determine corrosion rate (Vcorr) iron ASTM A36, in solutions of sodiumx cyanide and thiourea. Further evaluate the effect of this electrochemical phenomenon in costs associated with gold leaching processes. The corrosion rate was determined in $\mathrm{mm} /$ year by polarization curves. Costs were determined by a cost analysis using synthetic indicators. The corrosion rate of iron ASTM A36 in sodium cyanide solutions in concentrations of $0.62 \mathrm{~g} / \mathrm{L} ; 0.80 \mathrm{~g} / \mathrm{L}$ and thiourea solutions $0.96 \mathrm{~g} / \mathrm{L} ; 1.24 \mathrm{~g} / \mathrm{L}$ was evaluated. The results show that the corrosion rate of the metal is slightly higher in the solution of thiourea $1.24 \mathrm{~g} / \mathrm{L}(3.03$ $\mathrm{mm} /$ year) with the following behavior: Thiourea $1.24 \mathrm{~g}$ / L> Thiourea $0.96 \mathrm{~g} / \mathrm{L}>$ Cyanide $0.80 \mathrm{~g} / \mathrm{L} \approx$ Cyanide $0.62 \mathrm{~g} / \mathrm{L}$. It was estimated that corrosion costs in gold leaching processes are between 4.78 and $5.20 \%$ of the capital for cyanide and thiourea respectively.

Keywords: costs, corrosion, steel ASTM A36, hydrometallurgical processes.

\section{INTRODUCCIÓN}

La constante preocupación por conocer y solucionar los problemas operacionales ocasionados por el fenómeno de la corrosión, ha encaminado a diversas organizaciones e instituciones al desarrollo de investigaciones que permitan obtener un diagnóstico de las causas fundamentales que dan origen al problema (IONIS, 2012). Adicionalmente, el incremento de los costos de inversión y de mantenimiento, se ha convertido en el impulsor de las industrias para concentrar sus esfuerzos en recuperar energía, recursos y reducir los costos de sus procesos. Especialmente si están relacionados a las labores de mantenimiento preventivo o correctivo donde en gran medida y de forma errada se introducen los costos asociados a la corrosión. Esto no permite que las gestiones dirigidas a la optimación de recursos y minimización de costos se analice adecuadamente desde su origen y así, disminuir los mismo al mínimo conociendo las estrategias a emplear en materia de métodos de control (Aponte et al., 2008).

El impacto económico por la corrosión de los metales es medido en millones de dólares por año y se estima en aproximadamente el $4 \%$ del PIB (producto interno bruto) en los países industrializados (Cesàrea y Alcaraz, 2004). Por otro lado, la actividad minera en el Ecuador se encuentra en un estado de transición hacia la optimización ambiental de sus procesos, los mismos que tienen como necesidad implementar prácticas de tecnologías más limpias y mejorar la efectividad de los mismos. Las plantas de procesamiento aurífero de la Provincia de El Oro, en Ecuador, prestan servicios para el procesamiento hidrometalúrgico a las compañías con el fin de procesar las arenas. En ellas se pueden incluir las etapas de trituración y molienda, lixiviación por cianuración, flotación entre otras (Tello, 2015).

Durante el proceso de lixiviación, la disolución está regida por principios electroquímicos, Para llevar a cabo una lixiviación, es necesario que las fases sólida y líquida estén en íntimo contacto.

$1 \mathrm{PhD}$. Jefe Grupo de Investigación Aplicaciones Electroanalíticas Carrera de Ingeniería Química, Unidad Académica de Ciencias Químicas y de Salud; Universidad Técnica de Machala.

E-mail: hromero@utmachala.edu.ec

2 Ingeniera Química, Investigadora, Carrera de Ingeniería Química, Unidad Académica de Ciencias Químicas y de Salud; Universidad Técnica de Machala.

E-mail: dtellor_est@utmachala.edu.ec

$3 \mathrm{MgS}$ en Economía, Jefe Grupo de Investigación Producción de Biocombustibles, Unidad Académica de Ciencias Empresariales, Universidad Técnica de Machala.

E-mail: cvega@utmachala.edu.ec

4 Semillero de Investigación Aplicaciones Electroanalíticas, Carrera de Ingeniería Química, Universidad Técnica de Machala.

E-mail: aecastillos_est@utmachala.edu.ec 
De esta manera, la especie de interés se solubiliza transportándose de una fase hacia otra, separándose del material original (Mancheno, 2009).

Uno de los agentes lixiviantes más utilizados es el cianuro, el cual es considerado una de las sustancias tóxicas más peligrosas que se conoce en el mundo; por eso, en muchos países, su utilización ha sido prohibida. Es por esto que se han investigado agentes lixiviantes diferentes al cianuro, con el fin de utilizarlos como alternativas en los procesos de lixiviación de oro, entre los que se destaca la tiourea. Este agente lixiviante tiene el potencial para superar las limitaciones que presenta el uso del cianuro, su acción es menos afectada por la presencia de iones y especies inhibidoras, lo que conlleva a mayores cinéticas de lixiviación (Carrillo et al., 2013).

En este sentido, en el presente trabajo se evaluaron los costos por corrosión de tanques de lixiviación que utilizan como material el acero al carbono ASTM A36, con la finalidad de entregar un soporte técnico en el área de corrosión de equipos para un estudio de pre factibilidad técnico - económica, y evaluar un cambio en la tecnología hidrometalúrgica que representaría sustituir cianuro por tiourea como agente lixiviante del mineral aurífero.

\section{METODOLOGÍA}

Se prepararon soluciones de cianuro de sodio a concentraciones de 0,62 g / L; 0,80 g / L y de tiourea $0.96 \mathrm{~g} / \mathrm{L} ; 1.24 \mathrm{~g} / \mathrm{L}$, las cuales corresponden a las concentraciones con las que opera la planta de beneficio aurífero durante el proceso de lixiviación.

Se prepararon electrodos de trabajo a base del metal estudiado, con las siguientes dimensiones: 2.0 $\mathrm{cm} \times 2.0 \mathrm{~cm} \times 0.6 \mathrm{~cm}$ de espesor, recubriéndolos totalmente con resina epóxica, y dejando solamente expuesta un área de $1 \mathrm{~cm}^{2}$.

Para las pruebas electroquímicas se utilizó un Potenciostato Parstat MC con una celda en la que se dispone el metal a ser evaluado como electrodo de trabajo, un contra-electrodo de platino, un electrodo de referencia de $\mathrm{Ag} / \mathrm{AgCl}$ dentro de un capilar de Luggin; y como electrolito, soluciones de cianuro y tiourea en las concentraciones antes mencionadas. El experimento se realizó en modo potenciostático, para obtener las curvas de polarización (Sánchez, 2004). Conociendo que, las probetas son de acero al carbono y su potencial oscila alrededor de los $-0,76 \mathrm{~V}$ aproximadamente, se efectuó un barrido entre de $-2 \mathrm{~V} a+1,5 \mathrm{~V}$.

Adicionalmente, en los procesos electroquímicos, la velocidad de corrosión viene dada por la corriente de corrosión, por lo que, se utilizó el método de extrapolación de Tafel, y, mediante la "Ley de Faraday" se determinó la velocidad de corrosión (Jones, 1992). En este sentido, conociendo que la velocidad de corrosión se expresa como:

$$
\mathrm{V}_{\mathrm{corr}}=\frac{i m}{d z F}
$$

$$
\begin{aligned}
& \text { Dónde: } \\
& \mathrm{i}=\text { es la densidad de corriente en } \mathrm{A} / \mathrm{m}^{2} \\
& \mathrm{~m}=\text { peso atómico en } \mathrm{g} / \mathrm{mol} \\
& \mathrm{z}=\text { número de electrones } \\
& \mathrm{d}=\text { densidad del metal } \\
& \mathrm{F}=\text { constante de Faraday ( } 96500 \text { Coulumbs/equi- } \\
& \text { valente) }
\end{aligned}
$$

A partir de ello, se puede deducir la siguiente ecuación:

$$
\mathrm{V}_{\text {corr }}=0,0116 * \mathrm{i}_{\text {corr }}\left[\frac{\mathrm{mm}}{\mathrm{año}}\right]
$$

Esta ecuación se utilizó para calcular la velocidad de corrosión directamente a partir de la corriente de corrosión ( $i_{\text {corr }}$ ) obtenida por interpolación en las curvas de polarización.

En este estudio se utilizó el valor de la $\mathrm{i}_{\text {corr }}$ interpolada de la intersección de las pendientes anódicas y catódicas correspondiente al metal y en la respectiva solución lixiviante a la cual fue sometido el metal, conforme se puede observar en la Figura 1.

Así mismo, la valoración económica de los efectos del agente lixiviante sobre la infraestructura de la planta en el área de lixiviación, se realizó a través de un análisis de costos utilizando indicadores sintéticos de la corrosión y su impacto anual, frente al capital y proceso productivo. Los costos referentes al proceso de lixiviación, el capital y los rendimientos de oro procesado se estimaron en base a la información suministrada por la planta de beneficio aurífero, los mismos que, en detalle son confidenciales. Los resultados obtenidos fueron evaluados estadísticamente a través del uso del software libre R-project.

\section{RESULTADOS}

En la Figura 1 se presenta la curva de polarización del potencial (E) en función de la corriente (i) para el metal en una solución de cianuro $0.62 \mathrm{~g} / \mathrm{L}$ obtenida en los ensayos electroquímicos. En ella se puede 
observar, de manera demostrativa como, mediante la intersección de la interpolación de la pendiente de Tafel anódica y pendiente de Tafel catódica, se pudo obtener el valor de la corriente de corrosión. Así tenemos que, en esta Figura se obtiene una $i_{\text {corr }}$ de $239,8 \mu \mathrm{A} \mathrm{cm}^{-2}$, lo que reemplazando en la ecuación 2 permite determinar una velocidad de corrosión $\left(\mathrm{V}_{\text {corr }}\right)$ de 2,782 milímetro por año (mm/año).

Por su parte las Figuras 2 y 3 presentan una comparación entre las curvas de polarización de acero ASTM A36 en las soluciones lixiviantes menos concentradas y las más concentradas, es decir, cianuro $0.62 \mathrm{~g} / \mathrm{L}$ vs tiourea $0,96 \mathrm{~g} / \mathrm{L}$ y cianuro $0,80 \mathrm{~g} / \mathrm{L}$ vs tiourea $1,24 \mathrm{~g} / \mathrm{L}$ respectivamente.

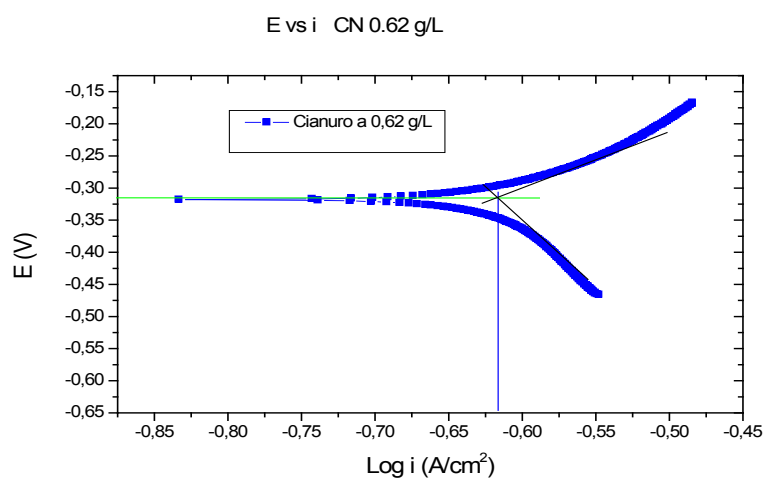

Fuente: Elaboración propia.

Figura 1. Curva de polarización para acero ASTM en cianuro 0,62 g/L, y determinación de la corriente de corrosión (icorr).

En la Tabla 1, se presentan los valores de $\mathrm{i}_{\text {corr }}, \mathrm{V}_{\text {corr }}$ y las pendientes de Tafel anódicas y catódicas obtenidos para todas las muestras metálicas en las soluciones lixiviantes evaluadas.

Tabla 1. Resultados de la corriente y velocidad de corrosion de acero ASTM A36 en las soluciones lixiviantes estudiadas.

\begin{tabular}{|l|c|c|c|c|}
\hline \multirow{2}{*}{$\begin{array}{c}\text { Concentracion } \\
\text { de la solución } \\
\text { lixiviante }\end{array}$} & \multirow{2}{*}{$\begin{array}{l}\mathbf{I}_{\text {corr }} \\
\left(\boldsymbol{\mu} \mathbf{A} / \mathbf{c m}^{2}\right)\end{array}$} & \multirow{2}{*}{$\begin{array}{c}\mathbf{V}_{\text {corr }} \\
(\mathbf{m m} / \mathbf{a n ̃ o})\end{array}$} & \multicolumn{2}{|c|}{$\begin{array}{c}\text { Pendientes de } \\
\text { Tafel }\end{array}$} \\
\cline { 4 - 6 } & & & Anódica & Catódica \\
\hline Cianuro 0,62 g/L & 239,8 & 2,782 & 0,62 & $-1,63$ \\
\hline Cianuro 0,80 g/L & 240,6 & 2,791 & 3,47 & $-1,55$ \\
\hline Tiourea 0,96 g/L & 253,6 & 2,942 & 0,78 & -2 \\
\hline Tiourea 1,24g/L & 261,3 & 3,032 & 1,13 & $-0,10$ \\
\hline
\end{tabular}

Fuente: Elaboración propia.

Los valores de velocidad de corrosion del acero obtenidos para el metal en las soluciones lixiviantes evaluadas en este estudio son de igual orden de magnitid a los obtenidos por otros autores en medio de cloruro de sodio ( $\mathrm{NaCl}$ ) al 3,5 \% (Molina, 2011), que han obtenido una velocidad de corrosion Vcorr de $2,020 \mathrm{~mm} /$ año en acero al carbono.

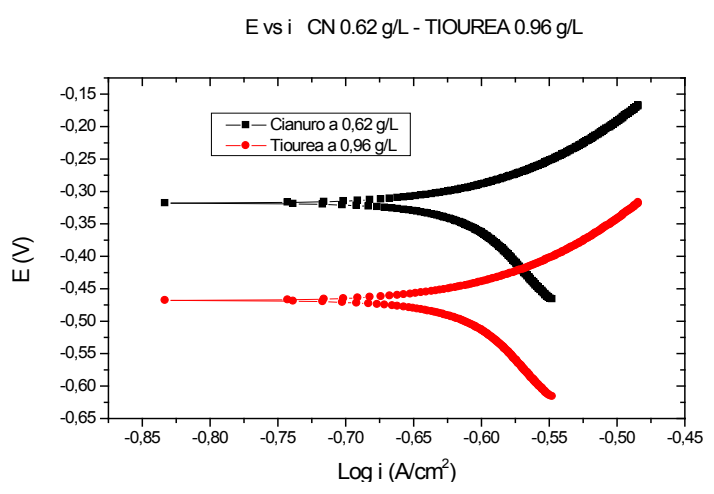

Fuente: Elaboración propia.

Figura 2. Curvas de polarizacion para acero ASTM A36 en cianuro $0,62 \mathrm{~g} / \mathrm{L}$ y Tiourea $0,96 \mathrm{~g} / \mathrm{L}$.

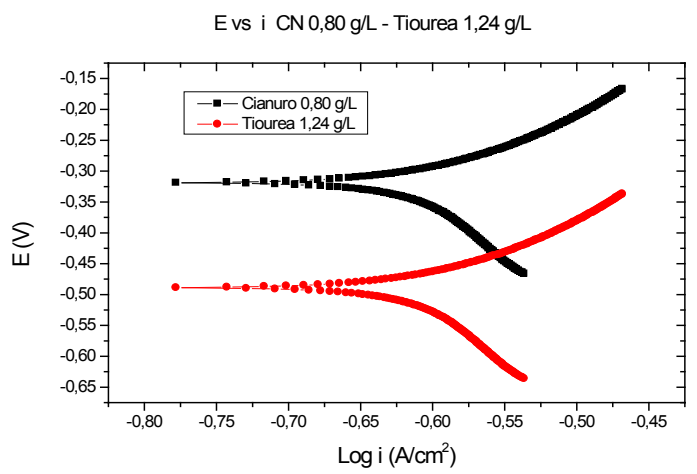

Fuente: Elaboración propia.

Figura 3. Curvas de polarizacion para acero ASTM A36 en cianuro 0,80 g/L y Tiourea 1,24 g/L.

Adicionalmente, la Tabla 2 presenta los costos por corrosión asociados al agente lixiviante en el procesamiento aurífero en términos anuales y considerando indicadores sintéticos referentes al capital y cotización actual de onza de oro procesado. Los costos anuales por corrosión estimados para el área de lixiviación en la planta de procesamiento aurífero se encuentran en un rango de entre \$ 19103.07 a \$ 20819.73 dólares por año equivalentes como porcentaje del capital determinado entre el $4.78 \%$ al $5.20 \%$. Estos valores obtenidos, son comparables a los reportados por Brongers \& Tubens 2001 , donde se indica que el costo promedio por corrosión en la industria química, petroquímica y farmacéutica es del $8 \%$ sobre el capital. 
Tabla 2. Costos asociados a la corrosión por tipo de agente lixiviante estudiado.

\begin{tabular}{|c|c|c|c|c|c|c|c|c|}
\hline $\begin{array}{c}\text { Agente } \\
\text { lixiviante }\end{array}$ & $\begin{array}{c}\text { Concentración } \\
(g / L)\end{array}$ & $\begin{array}{c}\text { Velocidad } \\
\text { de corrosión } \\
\text { (mm/año) }\end{array}$ & $\begin{array}{l}\text { Potencial de } \\
\text { corrosión } \\
\text { (V) }\end{array}$ & $\begin{array}{l}\text { Vida útil } \\
\text { tanque } \\
\text { (años) }\end{array}$ & $\begin{array}{l}\text { Costo por } \\
\text { corrosión } \\
\text { (\$/año) }\end{array}$ & $\begin{array}{l}\text { Costo total } \\
\text { por corrosión* } \\
(\$ / a n ̃ o)\end{array}$ & $\begin{array}{l}\text { Costos de } \\
\text { corrosión vs } \\
\text { Capital (\%) }\end{array}$ & $\begin{array}{l}\text { Costo de } \\
\text { corrosión } \\
\text { por onza de } \\
\text { oro }(\$ / O z)\end{array}$ \\
\hline Cianuro & 0.62 & 2.78 & -0.32 & 2.16 & 1910.31 & 19103.07 & 4.78 & 0.33 \\
\hline Cianuro & 0.80 & 2.79 & -0.32 & 2.15 & 1916.49 & 19164.87 & 4.79 & 0.33 \\
\hline Tiourea & 0.96 & 2.94 & -0.49 & 2.04 & 2020.17 & 20201.73 & 5.05 & 0.35 \\
\hline Tiourea & 1.24 & 3.03 & -0.47 & 1.98 & 2081.97 & 20819.73 & 5.20 & 0.36 \\
\hline
\end{tabular}

Planta de procesamiento aurífero con 10 tanques de proceso en área de lixiviación.

Fuente: Elaboración propia.

Se observa que, en términos absolutos, el uso de Tiourea como agente lixiviante frente al uso del Cianuro es ligeramente más corrosivo, con una diferencia entre el valor máximo y mínimo de $0.25 \mathrm{~mm} /$ año. La desviación estándar en los resultados obtenidos como porcentaje de la media en el conjunto de agentes lixiviantes analizados es del $4.21 \%$, lo que, en términos estadísticos y como se puede observar en la Tabla 3, esta diferencia no es significativa (p-value < 0.05). Es decir, con un 95\% de confianza se puede concluir que las velocidades de corrosión estimadas a diversas concentraciones para el cianuro y tiourea son estadísticamente similares.

Tabla 3. Resultados del test t para la media de una muestra.

\begin{tabular}{|l|}
\hline Prueba t, para una muestra \\
\hline Dato: Vcorr. mm/año. \\
$\mathrm{T}=47.516, \mathrm{df}=3, \mathrm{p}$-value $=2.052 \mathrm{e}-05$ \\
alternative hypothesis: true mean is not equal to 0 \\
Intervalo al $95 \%$ de confianza: \\
$2.693408 \quad 3.080092$ \\
Muestras estimadas: \\
Medias de $\mathrm{x}$ \\
$2.88675^{*}$ \\
\hline
\end{tabular}

Resultados obtenidos en el software libre R-project, paquete Rcomander. Fuente: Elaboración propia.

El costo de corrosión imputable por onza de oro procesada mostrado en la Tabla 2 varia del $0.33 \$$ / Oz a $0.36 \$ / O z$, lo que permite inferir que en términos de costos por corrosión a causa del agente lixiviante la Tiourea no genera diferencias significativas en el procesamiento aurífero, por lo tanto, la Tiourea es una alternativa económicamente viable en cuanto al impacto por corrosión, sin considerar los costos del proceso aurífero y los beneficios ambientales por la disminución en el riesgo ambiental consecuencia de que la Tiourea es menos contaminante que el Cianuro, más, esta línea de trabajo está pendiente para futuras investigaciones (Brongers y Tubens, 2001).

\section{CONCLUSIONES}

Los resultados muestran que la velocidad de corrosión del metal es ligeramente mayor en la solución de tiourea 1,24 g/L (3,03 mm/año) con el siguiente comportamiento: corrosión de hierro ASTM A36 en: Tiourea 1,24g/L > Tiourea 0,96 g/L > Cianuro $0,80 \mathrm{~g} / \mathrm{L} \approx$ Cianuro $0,62 \mathrm{~g} / \mathrm{L}$. Siendo importante mencionar que la corrosividad entre las dos concentraciones del lixiviante cianurado es aproximadamente similar.

Del análisis de costos por corrosión realizado en este estudio se puede concluir que el impacto en la inversión por corrosión en el área de lixiviación en el procesamiento aurífero comparando tecnologías de lixiviación por cianuro y tiourea se encuentran entre el $4.78 \%$ y el $5.20 \%$ con una media representativa del $4.96 \%$, lo que permite inferir un futuro prometedor para el desarrollo de tecnologías de lixiviación ambientalmente más amigables a partir de tiourea en el procesamiento aurífero.

\section{AGRADECIMIENTO}

Los autores agradecen a la Universidad Técnica de Machala y a FRANROMEC S.A. por el apoyo brindado durante el desarrollo de esta investigación.

\section{REFERENCIAS BIBLIOGRÁFICAS}

[1] Aponte et al, B. (2008). Corrosion costs in preventive and corrective maintenance in equipment and facilities in industry. Rev. Téc. Ing. Univ. Zulia, 87-96. 
[2] Brongers, M., \& Tubens, I. (2001). OALIB. Obtenido de Corrosion Cost and Preventive Strategies in the United States, Appendix V, Chemical, Petrochemical and Pharmaceutical; U.S. Department of Transportation, Federal Highway Administration: https://goo.gl/MvhCgA

[3] Carrillo et al, M. (2013). Liviviación con tiourea de dos minerales auroargentíferos santandeanos. Revista Colombiana de Materiales $N^{\circ} 5$, 319-324.

[4] Cesárea y Alcaraz. (2004). Manual básico de corrosion para ingenieros. España: EDITUM. Ediciones de la Universidad de Murcia.

[5] IONIS. (2012). Remando contra la corriente. Obtenido de: https://goo.gl/cdqSNb

[6] Jones. (1992). Principles and prevention of corrosion. New York, USA: Pearson -Prentice Hall, Upper Saddler River.

[7] Mancheno, M. y. (Mayo de 2009). Recuperación de oro de soluciones post-lixiviación con cianuro de sodio mediante cementación con cinc y adsorción en carbón activado. Recuperado el noviembre de 2015, de: https://goo. $\mathrm{gl} / 4 \mathrm{Q} \times 3 \mathrm{Gd}$

[8] Molina (2011). Estudio Avanzado de Corrosion: Análisis del estado de corrosión de un edificio modernista de Barcelona y estudio de mecanismos de protección basados en recubrimientos orgánicos. Universitat Politécnica de Catalunya,, 22-24.

[9] Sánchez. (09 de Octubre de 2004). Aplicación de técnicas electroquímicas en la evaluación de inhibidores de corrosion usados en la industria petrolera. 66-74. Venezuela: Tesis doctoral, Facultad de Ciencias.

[10] Tello, D. (2015). Estudio comparativo de la corrosión de materiales en acero en medios cianurados y de tiourea suceptibles al deterioro utilizando técnicas electroquímicas en la empresa minera FRANROMEC. CAMILO PONCE ENRÍQUEZ. 
